\title{
Application of Optical Flow Method for Imaging Diagnostic in JET
}

\author{
T. Craciunescu ${ }^{\mathrm{a}}$, A. Murari ${ }^{\mathrm{b}}$, A. Alonso ${ }^{\mathrm{c}}$, P.T. Lang ${ }^{\mathrm{d}}$, \\ G. Kocsis ${ }^{\mathrm{e}}$, I. Tiseanu ${ }^{\mathrm{a}}$, V. Zoita ${ }^{\mathrm{a}}$ and JET EFDA Contributors ${ }^{\S}$
}

JET-EFDA Culham Science Centre, Abingdon, OX14 3DB, UK.

(a) EURATOM-MEdC Association, National Institute for Laser, Plasma and Radiation Physics, Bucharest, Romania

(b) Consorzio RFX, Associazione EURATOM-ENEA per la Fusione, Padova, Italy

${ }^{(c)}$ Laboratorio Nacional de Fusion, EURATOM-CIEMAT, Madrid, Spain

(d) Association EURATOM-IPP, Max-Planck-Institut für Plasmaphysik, Garching, Germany

(e) RMKI-KFKI EURATOM Association, Budapest, Hungary

$\S$ See the Appendix of F. Romanelli et al., Fusion Energy Conference 2008 (Proc. 22nd Int. FEC Geneva, 2008) IAEA, (2008).

email:teddy@infim.ro

\section{Abstract}

An optical flow method is applied to the study of several fusion plasma relevant issues, including plasma wall interactions. A multi-resolution coarse-to-fine procedure is used in order to cope with large displacements of objects between consecutive frames, characteristic of plasma images captured by JET fast visible camera. Occlusion modeling is also implemented. The method is able to provide good results for JET fast-visible camera images which can be affected by saturation, discontinuous movement, reshaping of image objects, low gray-level in-depth resolution. Significant experimental cases concerning pellet injection, plasma filaments and MARFEs are analysed. The method is able to provide the real velocity for objects moving close to structures.

Keywords: tokamak, plasma diagnostics, image processing, optical flow. 


\section{Introduction}

A wide angle view fast visible camera (Photron APX) was recently installed in the Joint European Torus JET [1]. The camera is viewing the full poloidal cross-section of the vacuum vessel and is covering a toroidal extent of $\sim 90^{\circ}$. The wide angle view is appropriate for the study of pellet ablation, large scale instabilities and plasma wall interactions. Since the high confinement mode of operation (H-mode) is the standard operating regime envisaged for ITER, Edge Localised Mode instabilities (ELM) [2] are of particular importance considering the power loads they can induce on the plasma facing components. Recently it has been proven that the view of the fast visible camera is able to provide useful information about ELMs [3]. Investigations of pellet ELM triggering, as a method to mitigate the ELM-caused heat load on plasma facing components, have been also recently performed on JET by means of the fast visible camera [4]. The fast visible camera observations may support also the validation of existing theories concerning ELM energy transport [5] and the study of filamentary structures observed during the development of the ELM instabilities [6].

Important quantitative information can be obtained by applying image processing techniques to the data provided by the fast visible camera. Specifically, the sequences of ordered images allow the estimation of motion as either instantaneous image velocities or discrete image displacements, the so-called optical flow. The estimation of motion information from image sequences is a key problem in computer vision. The objective of the analysis consists of finding the vector field, which describes how the image is changing with time (see e.g. Refs. 7-8 for good overviews on this subject). This provides important information about the spatial arrangement of the objects viewed and the rate of change of this arrangement. Discontinuities in the optical flow can help in segmenting images into regions that correspond to different objects. Optical flow can be used also for object detection and tracking, as well as movement detection.

The aim of this paper is to prove that optical flow can be successfully applied to study different fusion plasma relevant issues, including plasma wall interactions, using images from the fast visible camera. It is proved that the optical flow approach is able to offer unique information concerning different plasma physical phenomena

\section{Overview of the Methods}

The optical flow method attempts to find the vector field which transforms one image into the next image in a sequence of frames. The most widely and accurately used differential methods can be classified into two large categories: i) local methods, such as the Lucas-Kanade technique [9], which assume a small neighbourhood of constant flow and which are more robust against noise and ii) global methods such as the Horn-Schunck approach [10] which introduce a regularization smoothness term, providing dense flow fields.

The basic assumption of both categories of techniques is that the grey levels of objects in subsequent frames do not change over time, which allows writing :

$$
f(x+u, y+v, t+1)-f(x, y, t)=0
$$


where $f$ is the intensity and the displacement field $(u, v)^{T}(x, y, t)$ is the optical flow. For small displacements the Taylor expansion can be used in order to obtain the following formulation of the optical flow constraint:

$$
f_{x} u+f_{y} v+f_{t}=0
$$

where subscripts denote partial derivatives.

The problem is prone to instabilities since small perturbations in the signal can create large fluctuations in its derivatives. A typical way to overcome this drawback consists of implementing image smoothing techniques in order to remove noise and to stabilize the differentiation process. The smoothing of the image sequence is performed prior to differentiation by convolving each frame with a Gaussian function:

$$
f^{(s m o o t h)}(x, y, t)=\left(K_{\sigma} * f\right)(x, y, t)
$$

where $\sigma$ is the standard deviation of the Gaussian $K_{\sigma}(x, y)$. The low-pass effect of the Gaussian convolution removes noise and other destabilizing high frequencies. Smoothing can be extended also to the temporal dimension. Of course the smoothing process must be used very carefully: smoothing must suppress noise but not eliminate useful information.

However, from the mathematical point of view, even after proper smoothing is applied, the problem (2) remains ill-posed: a single equation is not sufficient to uniquely compute the two unknowns $u$ and $v$ (the so-called aperture problem). In order to cope with the aperture problem, the Lucas-Kanade approach assumes that the unknown optical flow vector is constant within a neighbourhood of size $\rho$ (see again Ref. 9). Therefore $u$ and $v$ can be determined at the location $(x, y, t)$ from a weighted least square fit by minimising the function:

$$
E_{L K}(u, v)=K_{\rho} *\left(\left(f_{x} u+f_{y} v+f_{t}\right)^{2}\right)
$$

Using the notation:

$$
\begin{aligned}
& w \equiv(u, v, 1)^{T} \\
& |\nabla w|^{2}=|\nabla u|^{2}+|\nabla v|^{2} \\
& \nabla_{3} f=\left(f_{x}, f_{y}, f_{t}\right)^{T} \\
& J_{\rho}\left(\nabla_{3} f\right)=K_{\sigma} *\left(\nabla_{3} f \nabla_{3} f^{T}\right)
\end{aligned}
$$

the quadratic form (4) can be rewritten as follows:

$$
E_{L K}(w)=w^{T} J_{\rho}\left(\nabla_{3} f\right) w
$$

Minimizing (6) implies to solve the Euler-Lagrange system:

$$
\left\{\begin{array}{l}
\partial_{u} E_{L K}=0 \\
\partial_{v} E_{L K}=0
\end{array} \quad\left(\begin{array}{cc}
K_{\rho} * f_{x}^{2} & K_{\rho}^{*} f_{x} f_{y} \\
K_{\rho} * f_{x} f_{y} & K_{\rho} * f_{y}^{2}
\end{array}\right)\left(\begin{array}{l}
u \\
v
\end{array}\right)=\left(\begin{array}{l}
-K_{\rho} * f_{x} f_{t} \\
-K_{\rho} * f_{y} f_{t}
\end{array}\right)\right.
$$


A sufficiently large value for $\rho$ is very successful in rendering the method robust against noise. The problem remains severe in flat regions of the emission, where the image gradient vanishes and, consequently, the aperture problem persists and the method is unable to produce dense flow fields. This constitutes the major drawback of Lucas-Kanade approach and in general of local gradient methods.

In order to cope with this problem, global methods supplement the optical flow constraint with a regularising smoothness term. The optical flow $(u, v)$ is determined as the minimiser of the global energy functional:

$$
E_{H S}(u, v)=\int_{\Omega}\left(\left(f_{x} u+f_{y} v+f_{t}\right)^{2}+\alpha\left(|\nabla u|^{2}+|\nabla v|^{2}\right)\right) d x d y
$$

where $\alpha>0$ determines the amount of smoothness and serves as regularisation parameter: larger values for $\alpha$ result in a stronger penalisation of large flow gradients and lead to smoother flow fields. Minimising (8) leads to the Euler-Lagrange equations:

$$
\begin{aligned}
& 0=\Delta u-\frac{1}{\alpha}\left(f_{x}^{2} u+f_{x} f_{y} v+f_{x} f_{t}\right) \\
& 0=\Delta v-\frac{1}{\alpha}\left(f_{x} f_{y} v+f_{y}^{2}+f_{y} f_{t}\right)
\end{aligned}
$$

At locations with $\alpha|\nabla f| \approx 0$, no reliable local flow estimate is possible, but the regulariser $|\nabla u|^{2}+|\nabla v|^{2}$ fills in information from the neighbourhood (the so-called filling-in effect). Unfortunately global methods have been observed to be more sensitive to noise than local differential methods [11].

Using again the notations (5), relation (8) can be rewritten in the compact form:

$$
E_{H S}(w)=\int_{\Omega}\left(w^{T} J_{0}\left(\nabla_{3} f\right) w+\alpha|\nabla w|^{2}\right) d x d y
$$

Brun et al. [12] noticed the similarity between equation (6) of the Lucas-Kanade method and the first term under the integral in the formulation (9) of the Horn-Schunck method. This suggested a way to extend the Horn-Schunck functional to a combined local-global (CLG) functional, by replacing the matrix $J_{0}\left(\nabla_{3} f\right)$ with the structure tensor $J_{\rho}\left(\nabla_{3} f\right)$ [13] with some integration scale $\sigma>0$. The price paid for obtaining a combined local-global method is the much more complicated form of the Euler-Lagrange equations:

$$
\begin{aligned}
& 0=\Delta u-\frac{1}{\alpha}\left(K_{\rho} *\left(f_{x}^{2}\right) u+K_{\rho} *\left(f_{x} f_{y}\right) v+K_{\rho} *\left(f_{x} f_{t}\right)\right) \\
& 0=\Delta v-\frac{1}{\alpha}\left(K_{\rho} *\left(f_{x} f_{y}\right) u+K_{\rho} *\left(f_{x}^{2}\right) v+K_{\rho} *\left(f_{x} f_{t}\right)\right)
\end{aligned}
$$

It can be argued that the smoothness assumption in the quadratic optimization (9) does not respect discontinuities in the flow field. In order to be able to capture also locally non-smooth motion, it is necessary to allow outliers. This can be achieved by replacing $\alpha|\nabla w|^{2}$ in the second 
term of (9) with a non-quadratic penalizer: $\alpha \Psi_{2}\left(|\nabla w|^{2}\right)$. Outliers not only occur in the smoothness term but in the data term as well. It is easy to imagine situations where the optical flow constraint is not fulfilled, e.g. because of occlusion, brightness changes, noise. Consequently the non-quadratic penalizer becomes:

$$
E_{C L G}(w)=\int_{\Omega}\left(\Psi_{1}\left(w^{T} J_{\rho}\left(\nabla_{3} f\right) w\right)+\alpha \Psi_{2}\left(|\nabla w|^{2}\right)\right) d x d y
$$

The penalizer function $\Psi$ is chosen usually as $\Psi\left(s^{2}\right)=\sqrt{s^{2}+\varepsilon^{2}}$ [14]. The quantity $\varepsilon$ is not an additional parameter, but rather ensures the differentiability of $\Psi$ in $s=0$. It is chosen reasonably small, say $\varepsilon=0.001$. In general, nonlinear methods give better results at locations with flow discontinuities but require significantly larger computation time (see Refs. 7 and 15).

The sparse linear systems of equations (10) may be solved iteratively, usually by using the successive over-relaxation (SOR) method [16], which ensures a good compromise between simplicity and efficiency. SOR introduces an additional relaxation parameter $\omega \in(0,2)$; for $\omega=1$ one obtains the well-known Gauss-Seidel method.

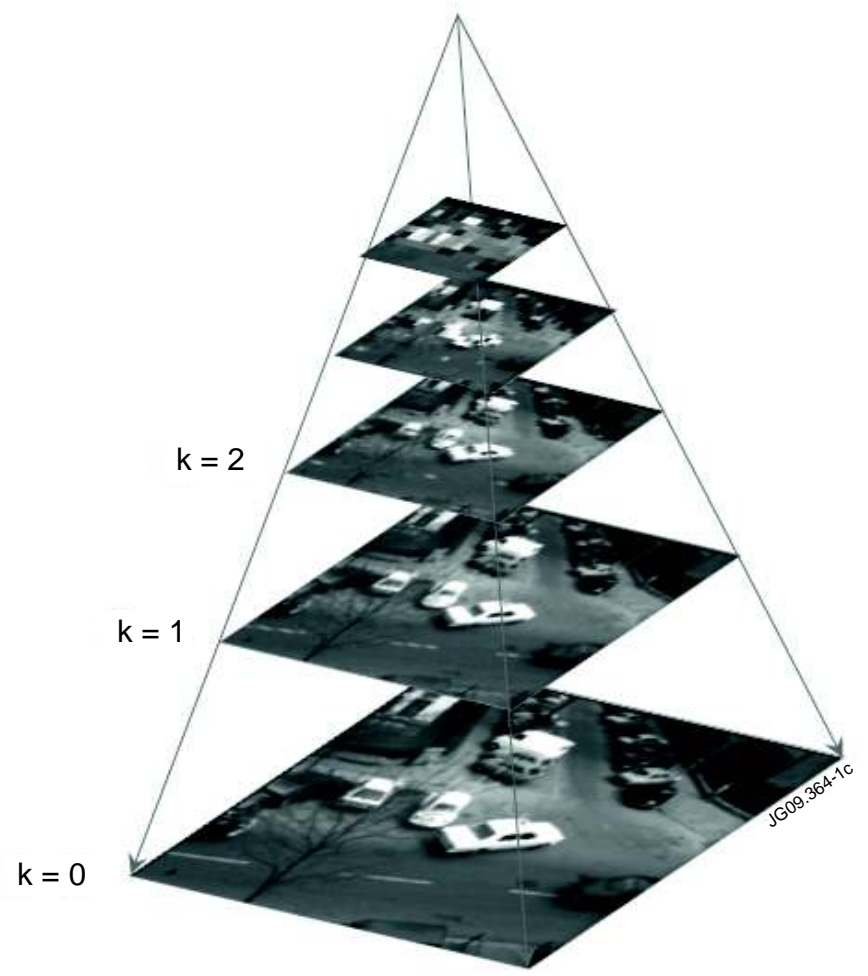

Figure 1 - The pyramid of images in the multi-resolution approach

In the case of displacements that are larger than one pixel per frame, typical of plasma images captured by JET fast visible camera, the cost functional in a variational formulation must be expected to be multi-modal and therefore a minimization algorithm can easily be trapped in a local minimum. In order to find the global minimum we used the multi-resolution coarse-to-fine 
procedure [17]. A pyramid of images $f_{k}$ is derived from the original frame $f$ by successive down-sampling and Gaussian smoothing steps (Fig. 1). Then the procedure starts at the coarse level, where the displacements $(u, v)$ are small and consequently the linearization of the grey value constancy assumption is a good approximation. This crude estimate is then refined step by step along the pyramidal structure. At resolution $k$, an increment velocity field $\left(d u_{k}, d v_{k}\right)$ is calculated around the estimate at the previous resolution level $k+1$, which is obtained by 'projecting' $\left(u_{k+1}, v_{k+1}\right)$ on the resolution frame $k$. This means to compensate the second image $f_{k}(\ldots, t+1)$ for the already computed flow field (the so-called warping step). The warping step is realized by using a backward registration approach that is based on bilinear interpolation. Since the increments $\left(d u_{k}, d v_{k}\right)$, corresponding to the finer resolution frame, are still small, the brightness constancy assumption is still valid. The final displacement field is obtained by a summation of all motion increments.

The calculated optical flow field may be affected by occlusions. Occlusions appear when two objects that are spatially separated in the $3 \mathrm{D}$ space might interfere with each other in the projected 2D image plane. Occlusion modeling is a difficult aspect of the optical flow. In this paper we have used the method suggested by Alvarez et al. [18] that simultaneously compute forward and reverse flow fields, labeling pixels as occluded where the two disagree. A bilateral filter, which incorporates flow from neighboring pixels that are similar in motion and appearance and that lie outside occluded regions, has also been used in order to improve boundaries [19]:

$$
u^{f i l t}(k)=\frac{\sum_{x_{j}, y_{j}} u\left(x_{j}, y_{j}\right) \cdot w\left(x . y, x_{j}, y_{j}\right)}{\sum_{x_{j}, y_{j}} w\left(x . y, x_{j}, y_{j}\right)}
$$

where:

$$
w\left(x . y, x_{j}, y_{j}\right)=\left\{\begin{array}{c}
K\left(\sqrt{\left(x-x_{j}\right)^{2}+\left(y-y_{j}\right)^{2}} ; \sigma_{x}\right) \\
K\left(I(x, y)-I\left(x_{j}, y_{j}\right) ; \sigma_{i}\right) \\
K\left(\sqrt{\left(u-u_{j}\right)^{2}+\left(v-v_{j}\right)^{2}} ; \sigma_{m}\right) \\
r\left(x_{j}, y_{j}\right)
\end{array}\right.
$$

An analogous expression can be written for $v$. The four terms in the filter expression (12) weight the neighbors according to spatial proximity, image similarity, motion similarity and occlusion labeling. 


\section{Experiments and Results}

The optical flow method has been implemented as described previously. The multi-resolution coarse-to-fine procedure was used in order to deal with large displacements. At each resolution level, the sparse linear systems of equations (10) were solved using the SOR method. After possible detection of occlusions, the velocity field images were filtered using the bilateral filter given by the relations $(12 \div 13)$. A large number of numerical tests have been performed on data already widely used as benchmarks to test the performance of this kind of image processing (see e.g. Ref. 20). A representative result is presented in Fig. 2 for the famous 'Yosemite' sequence with known velocities, available from $h t t p: / / w w w . c s . b r o w n . e d u / \sim b l a c k /$. The data was originally generated by Lynn Quam at the SRI International Artificial Centre (http://www.ai.sri.com/) and used for the first time by Heeger [21] for testing optical flow methods. The sequence was generated by taking an aerial image of Yosemite Valley and texture mapping it onto a depth map of the valley. A synthetic sequence was generated by flying through the valley. There are actually two common sequences: one with clouds and one without. The cloud pattern is fractal and undergoing Brownian motion. The assumption of brightness constancy does not hold for the clouds. Therefore Brown [22] recommends the use of the version without clouds which contains ground truth optical flow fields.
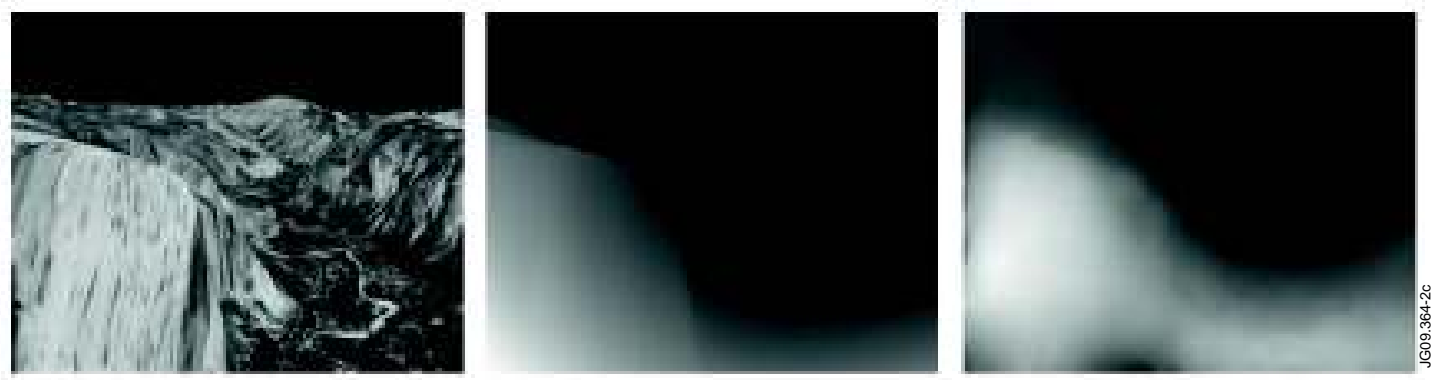

Figure 2 - Yosemite sequence: image 7 in the sequence (left), modulus of the velocities used to generate the transition from image 6 to image 7 (middle) and modulus of the reconstructed velocity field obtained by applying the optical flow method to the image pair image 6 -image 7 (right).

Fig. 2 reveals that the optical flow algorithm is able to retrieve the vector field with enough accuracy. The correlation coefficient between the image displaying the exact modulus $\sqrt{u^{2}+v^{2}}$ and the calculated one $\sqrt{u_{\text {calc }}^{2}+v_{\text {calc }}^{2}}$ is $\sim 0.83$ (this coefficient assumes the value 1 for a perfect matching of the two images).

Numerical tests have been necessary to determine the adjustable parameters of the algorithm: the parameter for Gaussian smoothing $\sigma$, the regularization parameter (smoothness term) $\alpha$, the regularization parameter for SOR algorithm $\omega$, the number of iterations in the SOR implementation, the number of levels of the images pyramid for multi-resolution implementation. The parameters of the algorithm depend on the structure of the image. However, a first approximation can be obtained using synthetic images.

Real images provided by the JET fast visible camera can be affected by saturation, discontinuous movement, reshaping of image objects, low gray-level in-depth resolution. 
Therefore, the basic assumptions of the optical flow model may not be verified and the algorithm will calculate an inaccurate vector field. Consequently a criterion to discern between correct and wrong calculated flow fields must be introduced. The optical flow is determined using two images $I_{1}, I_{2}$, from a sequence of images. Using the first image and the calculated optical flow, a version $I_{2}^{(\text {rec })}$ of the second image can be reconstructed. The similarity between $I_{2}$ and $I_{2}^{(\text {rec })}$ can be used in order to assess the accuracy of the optical flow calculations. Therefore the error image $I_{2}-I_{2}^{(\text {rec })}$ has been used as a confidence criterion to determine the validity of the results. An illustrative example is given in Fig. 3, for images taken from a sequence recorded with JET fast visible camera, showing ELM filaments moving up along the poloidal limiters. The confidence criterion is able to detect the failure of the optical flow calculations, determined by strong object reshaping and by the appearance of new objects in the second image.
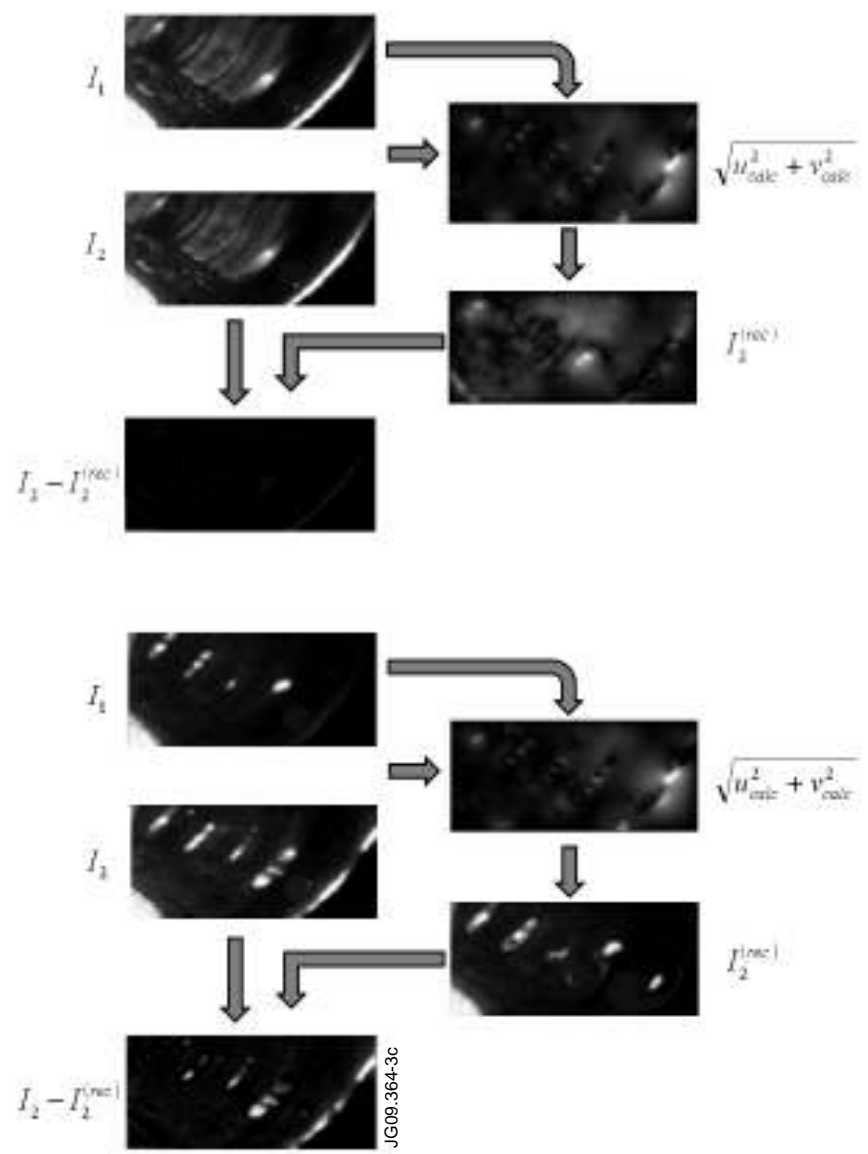

Figure 3 - Illustration of the confidence criterion. The error image can confirm the correct calculation of the optical flow field (top) but also emphasize wrong results, due to new objects appearing in the image sequence (bottom). The error image is represented, as the images in the sequence, on 64 gray-levels.

To validate the technique with experimental data, we have applied the optical flow method to determine the speed of pellets. In the case of the JET pulse \#76168, the pellet was emitted with a speed of $215 \pm 12 \mathrm{~m} / \mathrm{s}$ and it started to be recorded by the video camera as a visible object at $\mathrm{t}=20.011246$. At least at the beginning, for $\sim 40$ frames, the pellet is flying approximately on a straight line in the direction in which it was injected. This is indicated by the approximately 
fixed position of its reflection on the vacuum vessel components. Two frames from this sequence are presented in Fig. 4. As the direction of injection is known, together with the geometry of the experiment (see Fig. 5) it is possible to correlate the apparent speed of the pellet in the $2 \mathrm{D}$ images with its real speed in the $3 \mathrm{D}$ space. Later in the sequence, the radiation emitted by the pellet cloud determines an increased illumination of the scene (see again Fig. 5) which enables the identification of known objects in the scene such as the ICRH antenna. The presence of a known object in the image permits the conversion of length units from pixels to meters. The advantage of using the ICRH antenna for this conversion is that it is located in the centre of the camera field of view, in the neighbourhood of the position from which the pellet is emitted. The distance $\mathrm{AB}$ in Fig. 5 has 92 pixels which correspond to $1.4 \mathrm{~m}$.
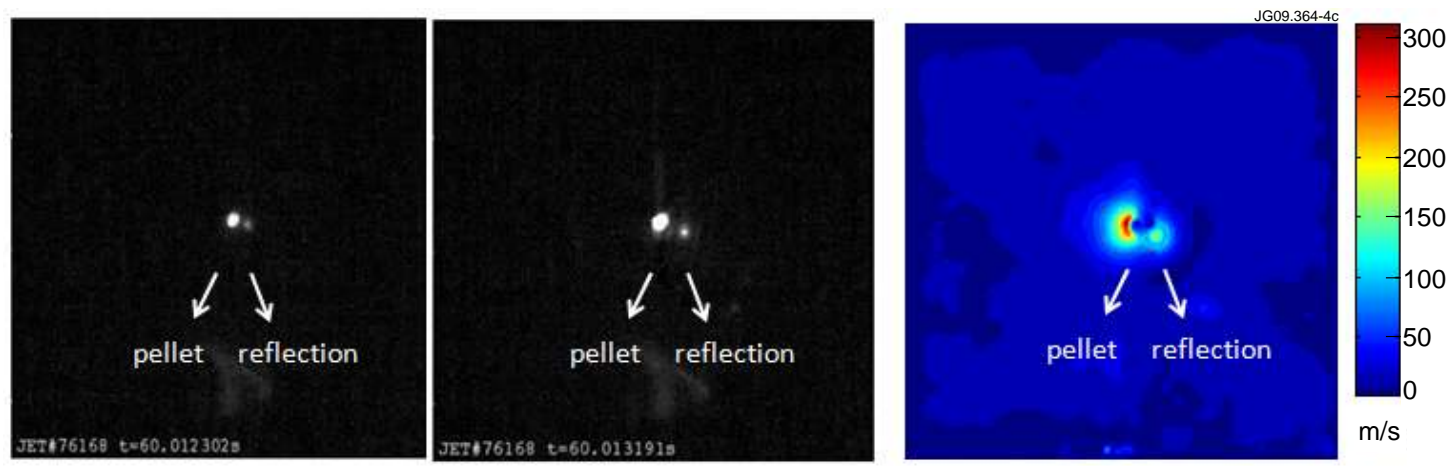

Figure 4 - Frames from the image sequence showing the pellet flying(left) - images are represented on 256 graylevels - and corresponding optical flow (right).

The optical flow has been calculated for different pairs of images selected from the part of the sequence that corresponds to the time interval when the pellet is flying approximately along a straight line. A representative result is presented in Fig. 4. It has been calculated using as input the images shown in the same figure. Two distinct shapes can be observed: one corresponds to the pellet and the other to the pellet mirror-image on the vacuum vessel which is also an object in the image. In principle, the flow field component corresponding to the mirror-image would not exist if the pellet was moving on a straight line. A slight deviation from this assumption, combined with the variation of the size of the bright area corresponding to the pellet (and consequently of its mirror-image) are responsible for the presence of this component. However its magnitude can be neglected as compared to the component corresponding to the pellet. Therefore the assumptions used for the pellet speed calculations are still valid.

Image segmentation has been used to isolate the component corresponding to the pellet movement. The speed has been determined as the average speed of the pixels of the flow field component corresponding to the pellet, and it has the value $\sim 270 \mathrm{~m} / \mathrm{s}$. The $\sim 25 \%$ error, relative to the speed of injection, erises from the uncertainty in measuring the distance AB in Fig. 5, the approximation related to the calculation of the projection of the pellet speed on the plane corresponding to the camera images, and also, as already mentioned, to the slight change of the size of the bright area corresponding to the pellet and a slight deviation from the assumption that the pellet is moving on a straight line. This result demonstrates the capability of the optical flow method to provide a reasonable estimate the speed of moving objects in the plasma. 
An interesting application of the optical flow method is the determination of the speed of plasma filaments. Plasma filaments are coherent structures characterized by enhanced visible radiation relative to the background plasma. Their motion is convective rather than diffusive, and they may differ from the background plasma in temperature and composition. Typically they are aligned with the magnetic field. The propagation is important to the overall dynamics in a variety of plasmas. In recent years, clear evidence of the ELM as a filamentary propagating structure have been shown. The interaction of ELM plasma filaments, with the tokamak first wall (divertor and limiter tiles) is one of the critical issues for ITER due to the deposited heat loads [23]. In several cases, the ELM filaments could be tracked along the surface of the outer limiters. Field-aligned plasma filaments are visible in the fast camera images as bright spots on the outboard poloidal limiters. A representative example is presented in Fig. 6. Images are recorded for JET pulse \#69903. The time separating consecutive frames is $33 \mu$ s and the integration time is $20 \mu \mathrm{s}$.

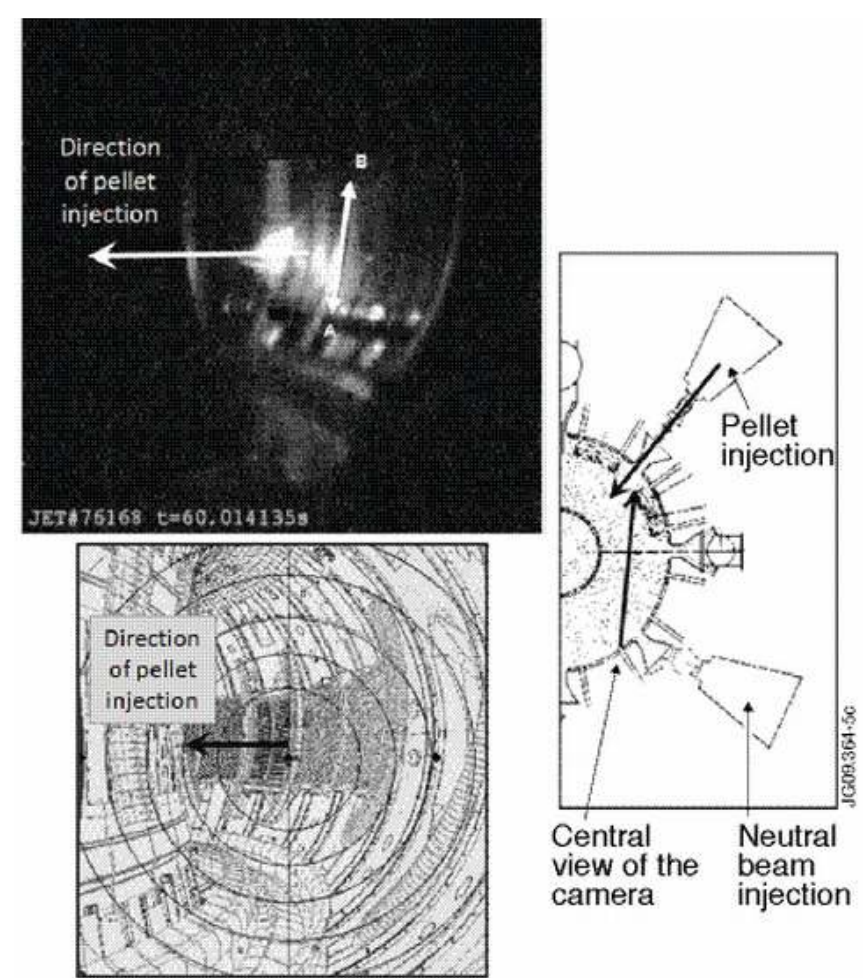

Figure 5 -Geometry of the experiment. A 3D view is obtained thanks to the illumination produced by the pellet (left-top); the direction of injection of the pellet is shown. The 3D information from the image can be correlated with geometrical information and with the camera view (left-bottom). The top view (right) permits the determination of the angle between the central view of the camera and the direction of injection of the pellet. The $3 D$ view permits the identification of components with known geometry as the ICRH antenna (segment AB). 

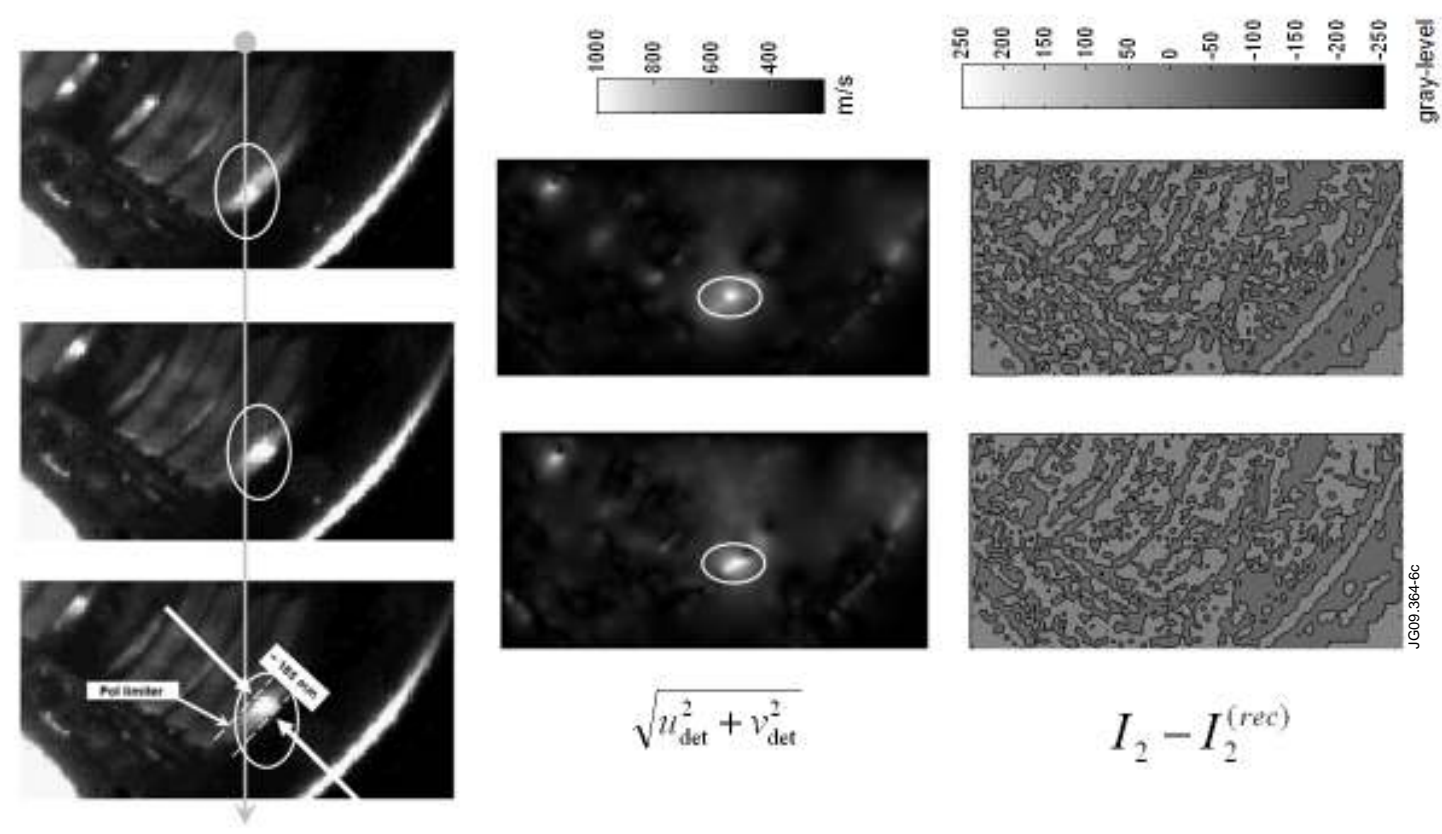

$$
I_{2}-I_{2}^{(\operatorname{rec})}
$$

Figure 6 - Image sequence showing ELM filaments moving up along the poloidal limiters (left), modulus of the optical flow field (middle) and error images (right). The moving filament for used for speed calculation is marked with a circle.

The application of the optical flow method is illustrated in Fig. 6, where the modulus of the velocity field and the error images are presented for a short sequence of three images. No pattern related to the moving filament can be observed in the error image, so the corresponding optical flow is accurately estimated. The low intensity structure of the error image is determined by several resizing and smoothing operations performed during the multi-resolution coarse-to-fine procedure. Using image segmentation techniques the region of interest corresponding to the moving filament has been extracted and used for speed calculations. The width of the poloidal limiter $(185 \mathrm{~mm})$, which corresponds to 14 pixels, has been used to convert pixels in length units. The optical flow calculations have been performed for a sequence of 10 images for which the basical assumptions of the method are fulfilled: no new objects appear in the image and objects reshaping can be neglected. The calculations have used as inputs both pairs of consecutive images and disparate images in the sequence. The calculated speed of the filament is $985 \pm 81 \mathrm{~m} / \mathrm{s}$.

The optical flow method has been applied also to determine the speed of MARFEs. MARFE is the acronym for "multi-faceted asymmetric radiation from the edge" and normally develops in fusion devices close to the density limit. MARFEs are considered the result of thermal instabilities excited under critical conditions through different mechanisms: impurity radiation, recycling of neutral particles, anomalous transport of charged particles and energy (see e.g. Ref. 24).

MARFEs appear as toroidally symmetric rings of enhanced radiation and usually occur on the high field side of the torus. A typical example is presented in Fig. 7, for the JET pulse \#70050. 
The time interval between two consecutive frames is $31 \mu \mathrm{s}$. The optical flow has been calculated for this sequence and the horizontal $u_{\text {calc }}$ and vertical $v_{\text {calc }}$ components, together with the modulus $\sqrt{u_{\text {calc }}^{2}+v_{\text {calc }}^{2}}$, are presented also in figure 7 . Image segmentation has been used to isolate the vector field corresponding to the MARFE and the speed has been calculated again as the average of the speed of the corresponding pixels. For pixels to meters conversion we have used the distance shown in Fig. 8 which is twice the minor radius of the torus.

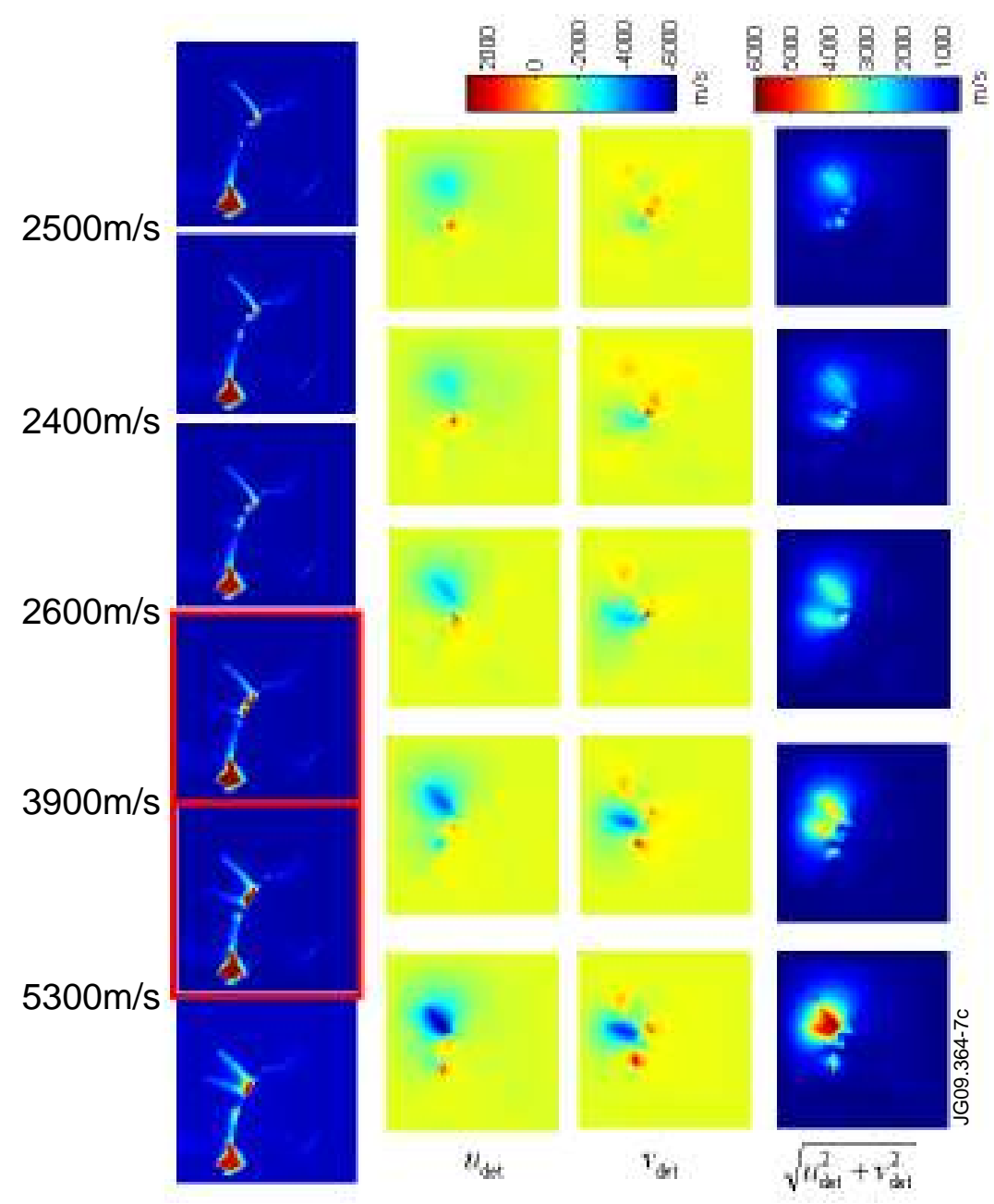

Figure 7 - MARFE image sequence (first column) and optical flow calculation: horizontal component (second second column), vertical component (third column) and modulus (last column). 

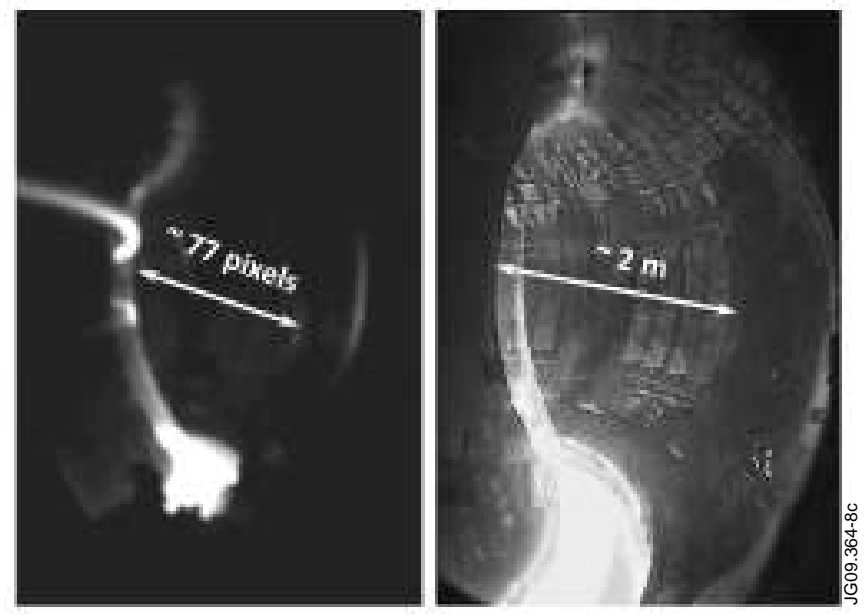

Figure 8 -Images used for the conversion of pixels to length units. The left image is from the MARFE sequence. The right one, taken from a different experiment, offer a better view of the details due to enhanced illumination.
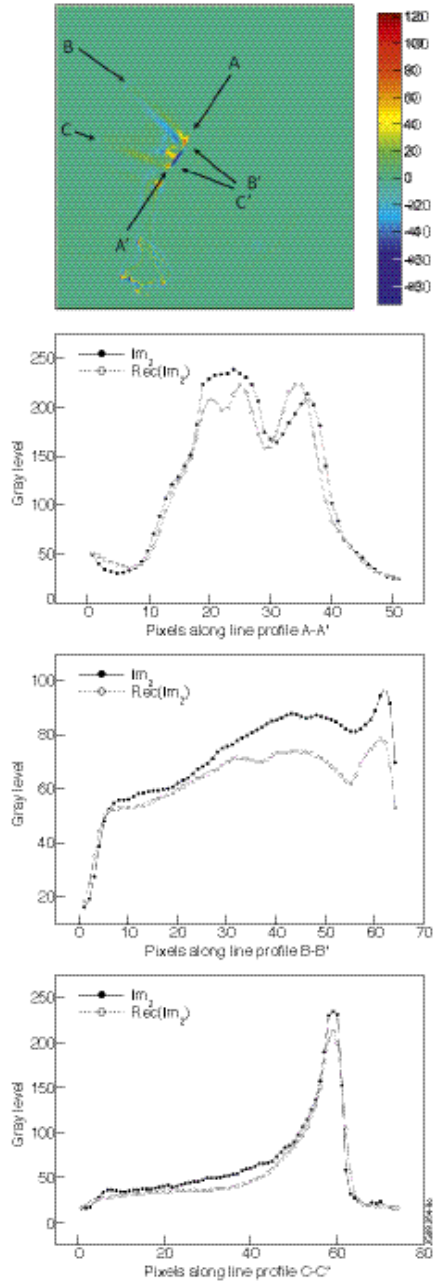

Figure 9 - Optical flow method applied to MARFEs. First row: the error image corresponding to the optical flow calculation for the fourth and fifth images in Fig.7; three lines $A A^{\prime}, B B^{\prime}$ and $C C^{\prime}$ are defined in order to calculated line profiles and to compare the second image $I_{2}$ with its reconstruction $I_{2}^{(\text {rec })}$. Second to fourth row: line profiles along the directions $A A^{\prime}, B B^{\prime}$ and $C C^{\prime}$ for second image $I_{2}$ (filled circles) and reconstruction of second image $I_{2}^{(\text {rec })}$ (empty circles). 
The error image corresponding to the optical flow between fourth and fifth images (marked with red border in Fig. 7) is presented in Fig. 9 (first row). In order to obtain a more complete picture about the accuracy of the calculated optical flow, three line profiles have been evaluated for the

second image $I_{2}$ and its reconstruction $I_{2}^{(r e c)}$ obtained from the first image $I_{1}$ and the calculated optical flow. The results are presented also in Fig. 9 (second to fourth row). The line profiles demonstrate that shapes are reconstructed accurately for all the three line profiles. Line profiles $\mathrm{BB}^{\prime}$ and $\mathrm{CC}^{\prime}$ are interesting from the point of view of MARFE speed calculation. In case of $\mathrm{CC}^{\prime}$ the reconstructed profile match, with a negligible error, the real one. In case of $\mathrm{BB}$ ' the shape is correctly reconstructed but a deviation of $\sim 25 \%$ arises in the second part of the profile. This indicate that if the speed is to be be calculated with higher accuracy, it is necessary to avoid this region or to use only the velocity field corresponding to CC'.

\section{Conclusion}

An algorithm based on the optical flow method, combining the advantages of local methods (robust under noise) and global techniques (which yield dense flow fields), is applied to JET fast visible camera image processing. The method incorporates a multi-resolution coarse-to-fine procedure in order to be able to work with large displacements between consecutive frames. Occlusion detection is also implemented. The method has been applied to the study of various issues relevant to the study of fusion plasmas: pellets, filaments, MARFEs. Extensively tested on synthetic images the method proved to achieve results in case of experimental images provided by the JET fastvisible camera which can be affected by saturation, discontinuous movement, reshaping of image objects, low grey-level in-depth resolution. For objects moving close to known structures of the tokamak vacuum vessel, the velocity in the real 3D space has been inferred from the 2D image analysis. The optical flow method is an unique tool able to cope with several different plasma physical phenomena and to provide useful information for modelling.

\section{Acknowledgements}

This work was supported by the European Communities under the contract of Association between EURATOM/MEdC, and was carried out within the framework of the European Fusion Development Agreement. The views and opinions expressed herein do not necessarily reflect those of the European Commission. 


\section{References}

[1] J. A. Alonso, P. Andrew, A. Neto, J. L. de Pablos, E. de la Cal, H. Fernandes, J. Gafert, P. Heesterman, C. Hidalgo, G. Kocsis, A. Manzanares, A. Murari, G. Petravich, L. Rios, C. Silva, P.D. Thomas, Fast visible camera installation and operation in JET, 34th EPS Conference on Plasma Phys. Warsaw, 2 - 6 July 2007 ECA Vol.31F, P-2.124 (2007).

[2] A. Kirk, H. R. Wilson, G. F. Counsell, R. Akers, E. Arends, S. C. Cowley, J. Dowling, B. Lloyd, M. Price, and M. Walsh, Spatial and Temporal Structure of Edge-Localized Modes, Phys. Rev. Lett. 92(2004)245002.

[3] J.A. Alonso, P. Andrew, A. Neto, J.L. de Pablos, E. de la Cal, H. Fernandes, W. Fundamenski, C. Hidalgo, G. Kocsis, A. Murari, G. Petravich, R.A. Pitts, L. Rios, C. Silva, Fast visible imaging of ELM-wall interactions on JET, Journal of Nuclear Materials, 390391(2009)797-800.

[4] G. Kocsis, J.A. Alonso, B. Alper, G. Arnoux, G. Cseh, J. Figueiredo, D. Frigione, L.Garzotti, J. Hobirk, S. Kalvin, M. Lampert, P.T. Lang, G. Petravich, T. Szepesi, R. Wenninger, Pellet cloud distribution and dynamics for different plasma scenarios in ASDEX Upgrade and JET, 36th EPS Conf. on Plasma Physics, Sofia, Bulgaria 2009, Europhysics Conference Abstracts, Vol. 33E, P-1.151, 2009

[5] R Wilson, S C Cowley, A Kirk, P B Snyder, Magneto-hydrodynamic stability of the Hmode transport barrier as a model for edge localized modes: an overview, Phys. Rev. Lett. 92 (24) (2004) 245002.

[6] W Fundamenski, R A Pitts, A model of ELM filament energy evolution due to parallel losses, Plasma Phys. Control. Fusion 48 (2006) 109-156.

[7] J. L. Barron, D. J. Fleet and S. S. Beauchemin, Performance of optical flow techniques, International Journal of Computer Vision, 12-1(1994)43-47.

[8] D. J. Fleet and Y. Weiss. Optical flow estimation. In Mathematical models for Computer Vision:The Handbook. N. Paragios, Y. Chen, and O. Faugeras (eds.), Springer, 2005.

[9] B. Lucas, T. Kanade, An iterative image registration technique with an application to stereo vision. In Proc. Seventh International Joint Conference on Artificial Intelligence, Vancouver, Canada, 1981, pp. 674-679.

[10] B. Horn, B. Schunck, Determining optical flow, Artificial Intelligence, 17(1981)185-203.

[11] A. Bruhn, J. Weickert, C. Schnörr, Combining the Advantages of Local and Global Optic Flow Methods, Proc. of 24th DAGM Symposium Zurich, Switzerland, September 16-18, 2002, in Lecture Notes in Computer Science, 2449(2002)454-462.

[12] A. Bruhn, J. Weickert, C. Schnorr, Lucas/Kanade meets Horn/Schunck: combining local and global optic flow methods, Int. J. Comput. Vision, 61-3(2005)211-231.

[13] J. Bigün, G.H. Granlund, and J. Wiklund, Multidimensional orientation estimation with applications to texture analysis and optical flow, IEEE Transactions on Pattern Analysis and Machine Intelligence, 13(8)(1991)775-790. 
[14] N.Papenberg, A.Bruhn, T.Brox, S.Didas, J.Weickert, Highly Accurate Optic Flow Computation with Theoretically Justified Warping, International Journal of Computer Vision, Vol. 67/2, 141-158, 2006.

[15] I. Cohen, Nonlinear Variational Method for Optical Flow Computation, Proceedings of the 8th Scandinavian Conference on Image Analysis, Tromso, Norway, MAY 25-28, 1993 pp. $523-530$..

[16] D.M. Young, Iterative Solution of Large Linear Systems. Academic Press: New York, 1971.

[17] E. Mémin, P. Pérez, Dense estimation and object-based segmentation of the optical flow with robust techniques. IEEE Transactions on Image Processing, 7-5(1998)703-719.

[18] L. Alvarez, R. Deriche, T. Papadopoulo, J. Sanchez, Symmetrical dense optical flow estimation with occlusion detection, In ECCV, pages 721.735, 2002.

[19] P. Sand, S.J. Teller, Particle Video: Long-Range Motion Estimation Using Point Trajectories. International Journal of Computer Vision 80-1(2008)72-91.

[20] H.H. Nagel, W. Enkelmann, W. An investigation of smoothness constraints for the estimation of displacement vector fields from image sequences, IEEE Transactions on Pattern Analysis and Machine Intelligence 8, 565-593, 1986. http://i21www.ira.uka.de/image_sequences/.

[21] D. J. Heeger. Model for the extraction of image flow, J. Opt. Soc. Am. A, 4(8):1455-1471, 1987.

[22] M. J. Black, A. Jepson, Estimating optical flow in segmented images using variable-order parametric models with local deformations, IEEE Transactions on Pattern Analysis and Machine Intelligence, 18-10(1996)972-986.

[23] G Federici, A. Loarte G. Strohmayer, Assessment of erosion of the ITER divertor targets during type I ELMs, Plasma Phys. Control. Fusion 45(2003)1523-1547.

[24] B. Lipschultz, B. LaBombard, E.S. Marmar, M.M. Pickrell, J.L. Terry, R. Waterson, S.M. Wolfe, MARFE: An Edge Plasma Phenomenon, Nucl. Fusion 24 (1984) 977. 